\title{
Investigation of reactions using polarized and unpolarized deuteron beam at Nuclotron: current status and perspectives
}

\author{
M. Janek ${ }^{1, \star}$, V.P. Ladygin ${ }^{2}$, V.V. Avdeichikov ${ }^{2}$, A.V. Averyanov ${ }^{2}$, A.S. Belov ${ }^{3}$, V.V. Fimushkin ${ }^{2}$, \\ Yu.V. Gurchin ${ }^{2}$, A.Yu. Isupov², J-T. Karachuk ${ }^{2,4}$, A.N. Khrenov², D.O. Krivenkov' ${ }^{2}$, A.K. Kurilkin², \\ P.K. Kurilkin ${ }^{2}$, N.B. Ladygina ${ }^{2}$, A.N. Livanov², G. Martinska ${ }^{5}$, S.M. Piyadin ${ }^{2}$, S.G. Reznikov², \\ G. Tarjanyiova ${ }^{1}$, A.A. Terekhin ${ }^{2}$, Ya.T. Skhomenko ${ }^{2,6}$, I.E. Vnukov ${ }^{6}$, and L.S. Zolin ${ }^{2}$ \\ ${ }^{1}$ Physics Department, University of Zilina, Univerzitna 1, 01001 Zilina, Slovakia \\ 2 Joint Institute for Nuclear Research, Joliot-Curie 6, 141980 Dubna, Moscow region, Russia \\ ${ }^{3}$ Institute for Nuclear Research of Russian Academy of Sciences, Moscow, Russia \\ ${ }^{4}$ Advanced Research Institute for Electrical Engineering, Splaiul Unirii 313, Bucharest, Romania \\ ${ }^{5}$ Institute of Physics, University of P. J. Shafarik, Park Angelinum 9, 04001 Kosice, Slovakia \\ ${ }^{6}$ Belgorod State University, Pobedy 85, 308015 Belgorod, Russia
}

\begin{abstract}
Deuteron induced reactions at intermediate energies are investigated at Internal Target Station (ITS) of Nuclotron. The $d p$ elastic scattering data have been accumulated in the angular range $\left(70^{\circ}-120^{\circ}\right)$ in c.m from 0.3 to $2.0 \mathrm{GeV}$ of deuteron energy. Part of the obtained results of differential cross sections and analyzing powers of $d p$ elastic scattering are compared with theoretical predictions based on relativistic multi-scattering model. The $d p$ breakup data are obtained with the detection of two outgoing protons at the angles of $\left(19^{\circ}-54^{\circ}\right)$ in laboratory frame at the deuteron energies from 0.3 to $0.5 \mathrm{GeV}$ in various detector configurations. One of the tools to study spin structure of short range correlations is the measurements of the cross section, tensor analyzing power $T_{20}$ and spin correlation parameter $C_{y, y}$ in the $d^{3} \mathrm{He} \rightarrow p\left(0^{\circ}\right)^{4} \mathrm{He}$ reaction at the energies between 1.0 and $1.75 \mathrm{GeV}$ using polarized ${ }^{3} \mathrm{He}$ target and extracted polarized deuteron beam from new polarized ion source at Nuclotron. For this purposes the feasibility study has been performed. Proposed experiments on extracted deuteron beam of Nuclotron is aimed to investigate: non-nucleonic degrees of freedom and baryonic resonances properties in the $\mathrm{d}\left(\mathrm{A}, \mathrm{d}\left(0^{\circ}\right)\right) \mathrm{X}$ and $\mathrm{d}\left(\mathrm{A}, \pi-\left(0^{\circ}\right)\right) \mathrm{X}$ reactions at different energies, non-nucleonic degrees of freedom via the measurements of the tensor $A_{y y}$ and vector $A_{y}$ analyzing powers in $\mathrm{d}(\mathrm{A}, \pi-) \mathrm{X}$ reaction and polarization properties of the baryonic resonances in the $\mathrm{d}(\mathrm{A}, \mathrm{d}) \mathrm{X}$ reaction and others. First measurement of vector and tensor polarization of new polarized ion source has been performed in June-July of 2016y.
\end{abstract}

\section{Introduction}

It is natural to describe few nucleon systems based on nucleon-nucleon $(\mathrm{NN})$ potentials. There are two approaches how the NN potentials are constructed. Realistic potentials as AV18 [1], CD-Bonn [2] and Nijm I, II and 93 [3] are based on meson-exchange picture. Effective field theories are based on

^e-mail: janek@fyzika.uniza.sk 
spontaneously broken chiral symmetry [4]. These realistic NN potentials and effective field theories describe NN data with very high accuracy. Many experimental and theoretic studies are aimed on the lightest nuclei in the intermediate energy region below the pion threshold. The advantage of this energy region is that the exact calculations can be done. Nowadays, Faddeev equations for all NN potentials can be solved exactly. But these realistic NN are unable to describe binding energy of three [5] and four nucleon systems [6] and many scattering experiments. Cross sections and the tensor analyzing powers obtained from $N d$ scattering at energies below $30 \mathrm{MeV}$ are well described with the corresponding theoretical calculations in which only the NN potentials have been used. In case of the vector analyzing power $A_{y}$ such an agreement couldn't be achieved using any known NN potential. The inclusion of the three nucleon force (3NF) did not improve the situation leading to the well known $A_{y}$ puzzle. The most used contribution in theoretical models which included $3 \mathrm{NF}$ are two pion exchange between all three nucleons. This contribution has been proposed long ago by Fujita-Miyazawa [7]. Idea of two pion exchange has been adopted and further developed in models of Urbana IX [8] and Tucson-Melbourne [9]. The success has been demonstrated in descriptions of binding energies of three and four nucleon systems. The cross section data of the $p d$ elastic scattering obtained at $250 \mathrm{MeV}$ cannot be reproduced by the Faddeev calculations [10]. Existence of new type of short range $3 \mathrm{NF}$ could be the reason for observed discrepancy. It has been found that in the vicinity of the Sagara discrepancy the currently known $3 \mathrm{NF}$ contribute by up to $30 \%$ to the $d p$ elastic scattering cross section at intermediate energies [11]. The investigation of $d p \rightarrow p p n$ reaction at deuteron energy of $270 \mathrm{MeV}$ at RIKEN [12] and IUCF [13] reveals variation in contribution of NN and 3NFs in polarization observables. Vector analyzing power $A_{y}$ can be described using only NN forces. Similar picture has been observed in KVI in $d p$ breakup reaction at energy of $130 \mathrm{MeV}$ [14]. Inclusion of $3 \mathrm{NF}$ improves a part of the polarization data but breaks other. The spin structure of the $n p$ SRCs has been investigated at JINR via the measurements of the tensor analyzing power $A_{y y}$ in deuteron inclusive breakup at different energies in the wide regions of the $x_{F}$ and transverse proton momentum $p_{T}$ [15]. Obtained results were compared with calculations using standard [16] and covariant deuteron wave functions [17], respectively. The $A_{y y}$ data clearly demonstrate the dependence on two internal variables, $x_{F}$ and $p_{T}$ (or their combinations). However, the use of the deuteron structure function that depends on two variables [17] does not allow to describe the data. Few nucleon scattering has a rich phase space. Variety of polarization observables can be measured under different kinematic conditions. The comparison of polarization observables obtained in various parts of phase space with theory can give information about dynamics beyond pairwise potential, Coulomb and relativistic effects and their relative strength.

\section{Experiments at ITS of Nuclotron}

The goal of the Deuteron Spin Structure (DSS) experimental program is to obtain the information about two and $3 \mathrm{NFs}$, including their spin dependent parts from $d p$-elastic scattering at the energies between $0.3-2.0 \mathrm{GeV}$ and $d p$-breakup with registration of two protons at deuteron energies of 0.3 $-0.5 \mathrm{GeV}$ [18]. Deuterons are accelerated by linear accelerator and Nuclotron up to required energy (see figure 1). Polyethylene and Carbon targets enclosed in a spherical hull of Internal Target Station (ITS) [19] were used. $D p$ effect is obtained by subtracting Carbon contribution from Polyethylene spectra. Different detector setups were used for $d p$ breakup and elastic scattering reaction investigations. 

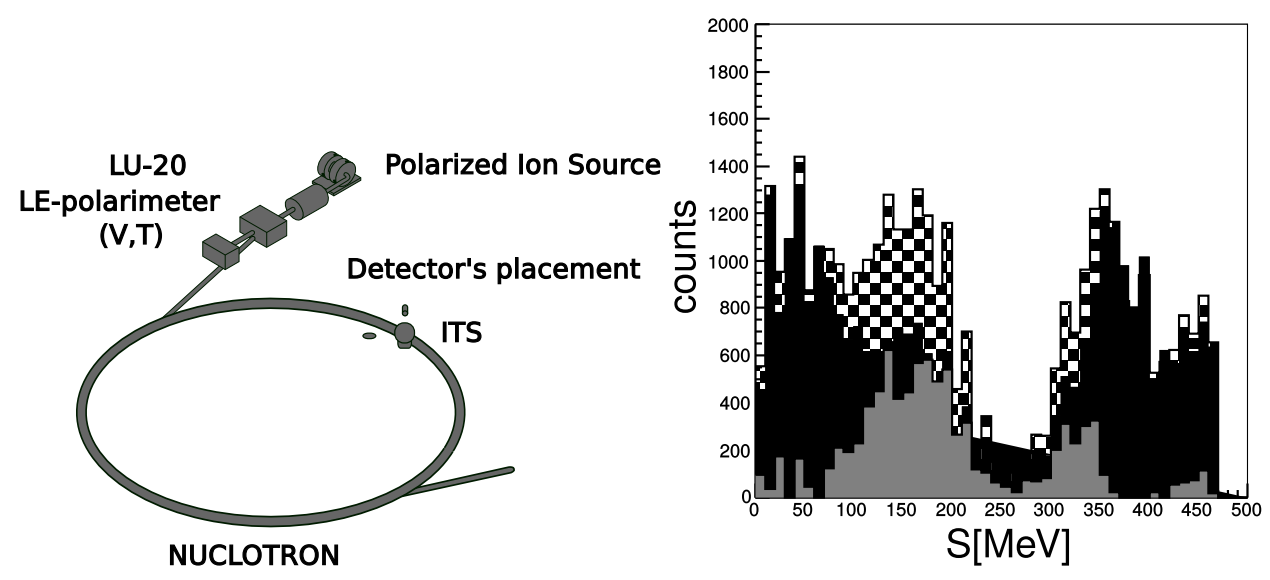

Figure 1. Left panel - Sketched view of Nuclotron accelerator with marked positions of ITS, detector setup for $d p$ elastic and $d p$ breakup reaction investigations, ion source and low energy polarimeter. Right panel - checked, black and grey histograms represent preliminary results of $S$ distribution obtained on Polyethylene, Carbon and subtracted $(d p)$ one for the detector arms placed at angles of $\Theta_{1}=35.0^{\circ}, \Theta_{2}=43.0^{\circ}$ and $\Phi_{12}=180^{\circ}$ at deuteron energy of $0.4 \mathrm{GeV}$.

\subsection{Dp breakup reaction}

The $d p \rightarrow p p n$ breakup reaction is investigated by $\Delta E-E$ scintillation detectors. The deuteron energies are $0.3,0.4$ and $0.5 \mathrm{GeV}$. Up to eight $\Delta E-E$ detectors can be used in experiment. Each detector consists of thin and thick scintillator. Both scintillators are of a tube shape, the thin one of a height of $1 \mathrm{~cm}$, the thick one of a $20 \mathrm{~cm}$ height, with the diameter of the cross section $8 \mathrm{~cm}$ and $10 \mathrm{~cm}$, respectively. Two Photomultiplier tubes PMTs- 85 are positioned opposite to each other at the outside cylindrical surface of the thin scintillator. At the bottom end of the $E$ scintillator a photomultiplier tube PMT-63 is positioned. Solid angle of each of detector is $4.6^{\circ}$. The details of the $\Delta E-E$ detector construction can be found in [20]. Energy spectra and missing mass distributions of $d p$ breakup reaction were compared with GEANT4 simulation [21]. Reasonable agreement gives us opportunity to handle the signal and background in more precise way. The GEANT4 modelling also confirms that energy losses and scattering angles of the particles which passed through the spherical steel hull that surrounds the target are relatively small except of region of very low energy up to 20 $\mathrm{MeV}$. But protons with this energy energy are cut by coincidence condition of $\Delta E-E$ detector.

Special setup of the detectors has been chosen in the experiment performed at 2014 to follow inverse kinematics of the reaction in which relativistic effects can play a role [22]. The kinematic variable $S$ corresponds to the arc-length along the kinematic curve with zero point chosen at the minimal value of deposited energy in the second arm. Points of kinematic $S$-curve are calculated with energy step of $5 \mathrm{MeV}$ in case of $d p$ breakup reaction for the detector arms placed at polar angles of $\Theta_{1}=35.0^{\circ}, \Theta_{2}=43.0^{\circ}$ and $\Phi_{12}=180^{\circ}$ (azimuthal angle between arms) at deuteron energy of $0.4 \mathrm{GeV}$. The $S$ is increasing in anti-clockwise direction. The number of breakup events in an interval $S-\Delta S / 2$, and $S+\Delta S / 2$ was obtained by projecting the events on a line perpendicular to the $S$ - 


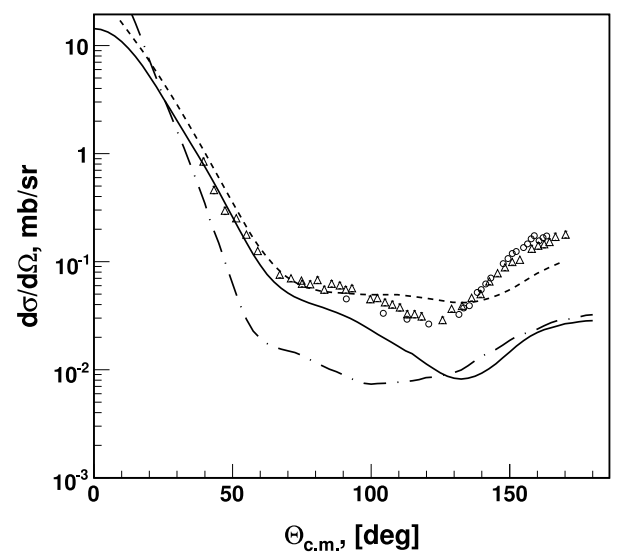

Figure 2. The angular dependence of the differential cross section obtained at $940 \mathrm{MeV}$ [24] and $850 \mathrm{MeV}$ [25] are represented by the open circles and triangles, respectively. The solid, dashed and dot-dashed lines are the results of the non-relativistic Faddeev calculations [26], relativistic multiple scattering model [27, 28] and optical potential approach [29].

curve. Preliminary result for energy step of $\Delta S=5 \mathrm{MeV}$ and for the arm's polar angles of $\Theta_{1}=35^{\circ}$, $\Theta_{2}=43^{\circ}$ and azimuthal angle between them $\Phi_{12}=180^{\circ}$ for Polyethylene (checked spectra), Carbon (black spectra) and subtracted (grey spectra) at deuteron energy of $0.4 \mathrm{GeV}$ is presented on the right panel in figure 1. Carbon normalization was performed on the parts of Polyethylene spectra where only Carbon content is assumed.

\subsection{Dp elastic scattering}

The results on the angular dependencies of the analyzing powers $A_{y}, A_{y y}$ and $A_{x x}$ of $d p$ - elastic scattering have been obtained at Nuclotron at $880 \mathrm{MeV}$ [23]. The differential cross section obtained at $940 \mathrm{MeV}$ [24] and $850 \mathrm{MeV}$ [25] in the earlier experiments are shown in figure 2 by the open circles and triangles, respectively. The solid, dashed and dot-dashed lines are the results of the nonrelativistic Faddeev calculations [26], relativistic multiple scattering model [27, 28] and optical potential approach [29]. The Faddeev calculations without invoking $3 N$ forces reproduce the behaviour of the analyzing powers, however, they fail to describe the cross section data at the scattering angles larger than $70^{\circ}$ in the centre of mass system. The calculations performed within relativistic multiple scattering model $[27,28]$ describes the data on the vector analyzing power $A_{y}$ and cross section. However, there are some problems in the description of the tensor analyzing powers at large angles in the centre of mass system. The optical potential approach fails to reproduce both cross section and analyzing powers. The observed deficiencies in the description of the differential cross section and deuteron analyzing powers at $T_{d} \sim 880 \mathrm{MeV}$ obtained at quite large transverse momenta require the consideration of the additional mechanisms, for instance, $3 N$ forces. Since present $3 N$ forces models cannot improve the agreement with the data obtained even at lower energies, new models of $3 \mathrm{~N}$ forces (including their short-range part) should be considered. For instance, the next step in the relativistic multiple scattering model $[27,28]$ development could be taking into account the explicit $\Delta$ - isobar excitation. 

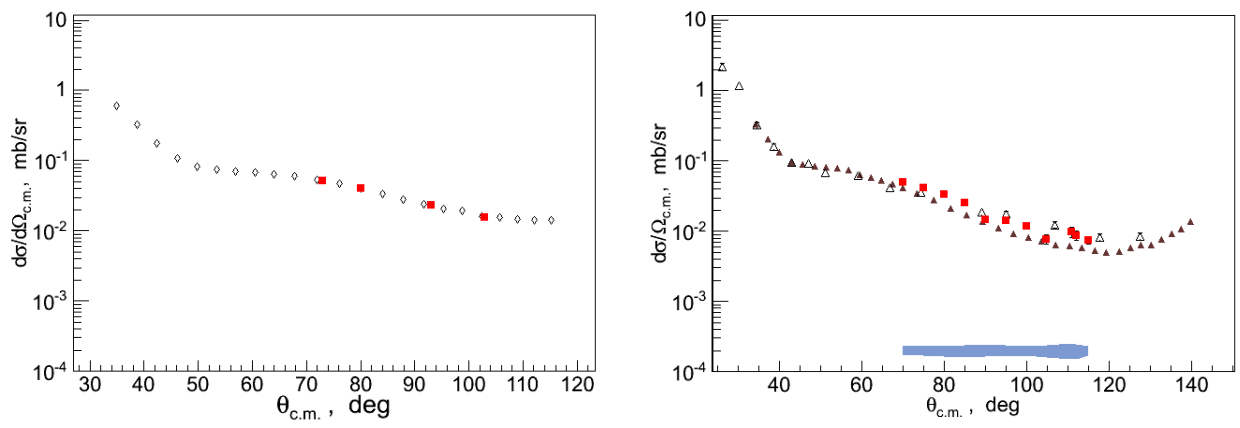

Figure 3. The angular dependence for differential cross section at $650 \mathrm{MeV} / \mathrm{n}$ (left panel) and $700 \mathrm{MeV} / \mathrm{n}$ (right panel). Rhombuses represent data obtained at $641.3 \mathrm{MeV} / \mathrm{n}$ [30]. Solid and open triangles represent data from $800 \mathrm{MeV} / \mathrm{n}$ [31] and $794 \mathrm{MeV} / \mathrm{n}$ [30], respectively. Red squares are data obtained at Nuclotron. Their systematic errors are shown by the solid blue band.

$D p$ elastic data have been obtained in the energy range from 0.3 to $2.0 \mathrm{GeV}$. Presented $d p$ elastic data were obtained by high energy $d p$ detector setup. Firstly, the normalization was performed at the energy of $650 \mathrm{MeV} / \mathrm{n}$. The angular dependence of differential cross section [30] was approximated by the exponential function in angular range $71^{\circ}<\theta_{c . m}<105^{\circ}$. Obtained normalization was used to obtain the differential cross section for other energies. Angular dependence of differential cross section at $650 \mathrm{MeV} / \mathrm{n}$ and $700 \mathrm{MeV} / \mathrm{n}$ are shown on the left and right panels of figure 3. The systematic errors are shown by the solid blue band.

\section{Experiments at extracted deuteron beam}

Transportation line of the Nuclotron extracted beam to the start version of BM@N spectrometer is shown in figure 4 . Spin physics with extracted polarized deuteron beam from new polarized deuteron source [32] can be performed with the start version of the BM@N setup [33]. The BM@N experimental setup will be installed at the renovated $6 \mathrm{~V}$ beamline [34] in the fixed-target hall of the Nuclotron. The $6 \mathrm{~V}$ beamline contains the quadrupole lenses doublet, two dipole magnets allowing to correct the beam position in the vertical and horizontal planes, and SP-41 dipole magnet with enlarged aperture for the momentum measurements [35].

Spin program realization requires to install the polarimeters (with $\mathrm{CH}_{2}-\mathrm{C}$ targets) at F3 and F5 focuses for polarimetry of the deuteron (or proton) beam. Liquid hydrogen (deuterium) target, polarized ${ }^{3} \mathrm{He}$ target [36] and set of nuclear targets is needed to place at F5 or F3. The spin physics with the target position at F5 (or F3) focus is related with the measurements of the polarization observables in the reactions with the emission of the detected particle at forward angles. The solid angle is defined by the lenses doublet $7 \mathrm{k} 100-8 \mathrm{k} 100$ of the VP1 transportation beam line.

\section{$3.1 d\left({ }^{3} \mathrm{He}, p\right)^{4} \mathrm{He}$ reaction}

The goal of the ${ }^{3} \mathrm{He}(d, p)^{4} \mathrm{He}$ reaction study at Nuclotron is to understand the reasons of strange structure in the behaviour of the tensor analyzing power $T_{20}$ in $d p$ - backward elastic scattering [38, 39] 


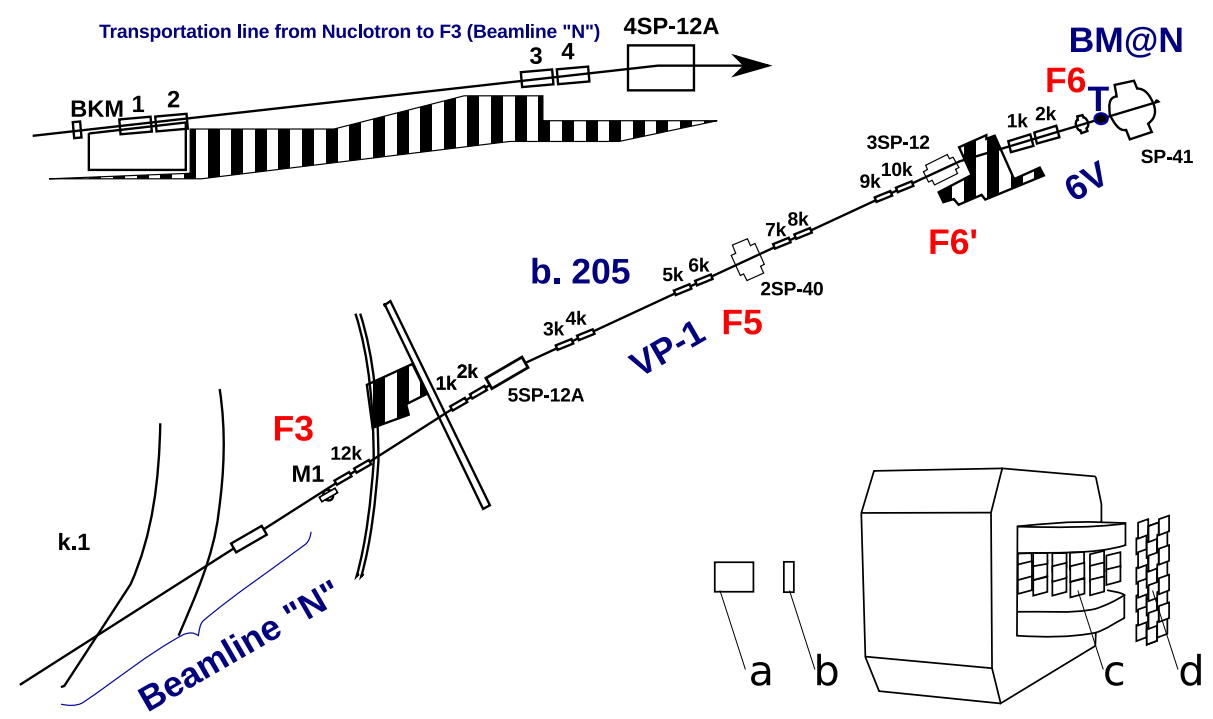

Figure 4. Transportation line of the Nuclotron extracted beam to the BM@N spectrometer with start version of BM@N setup (bottom right). Spin physics with BM@N can be associated with different target positions: at F5 (F3), F6' and F6. Schematic view of the part of BM@N setup used in simulation is shown in the right-down corner; a - ${ }^{3} \mathrm{He}$ target, b - start counter (quartz radiator), c - 12 GEM tracker stations, d - TOF wall.

at the internal momentum $k \sim 0.3-0.5 \mathrm{GeV} / c$ in the vicinity of the $D$ - wave dominance. The experiments performed at RIKEN at the energies below $270 \mathrm{MeV}$ have shown that the polarization correlation coefficient $C_{/ /}$for the ${ }^{3} \mathrm{He}(d, p)^{4} \mathrm{He}$ reaction may be a unique probe to the D-state admixture in deuteron [40] due to the strong spin-selectivity in neutron capture process by ${ }^{3} \mathrm{He}$ nucleus. In the one-nucleon exchange (ONE), the expression for $C_{/ /}$is proportional to the $\mathrm{D}$-state fraction in deuteron $T_{20}$ and $\kappa_{0}$ for $d p$ backward elastic scattering which include $S$ - and D-state interference term together with a squared $\mathrm{D}$ contribution. Thus expected that $C_{/ /}$may be a candidate to provide an information on the deuteron structure complementary to those from $T_{20}$ and $\kappa_{0}$ obtained in $d p$-backward elastic scattering [38].

The main goal of the proposed experiment is to obtain the data on $C_{/ /}$in the energy region of 1.0-1.75 GeV, where the contribution from the deuteron D-state is expected to reach a maximum in one-nucleon exchange approximation, to obtain new information on the strange structure observed in the behaviour of $T_{20}$ in the $d p$ - backward elastic scattering and to realize experiment on the full determination of the matrix element of the ${ }^{3} \mathrm{He}(d, p){ }^{4} \mathrm{He}$ reaction in the model independent way. These data will help us also to understand the short-range spin structure of deuteron and effects of non-nucleonic degrees of freedom. For these purposes polarized deuteron beam from new PIS [32] and spin-exchange-type polarized ${ }^{3} \mathrm{He}$ target developed at CNS of Tokyo University [36] and modified for the experiment at Nuclotron can be used. Experiment is planned to be carried out with extracted deuteron beam of Nuclotron at the BM@N area at energy range of $1.0-1.75 \mathrm{GeV}$. The feasibility study of the $d^{3} \mathrm{He} \rightarrow p^{4} \mathrm{He}$ reaction have been investigated using GEANT4 and ROOT simulation package [37]. There are serious requests on beam size and emittance in the target crossover point. Deuteron beam hits the ${ }^{3} \mathrm{He}$ target which is placed in the one of the focuses of the beam transportation line of Nuclotron, F5 focus point is assumed in next. The length of polarized ${ }^{3} \mathrm{He}$ target is $30 \mathrm{~cm}$. Scattered protons and deuterons, as well as primary deuterons pass along transportation beam line 

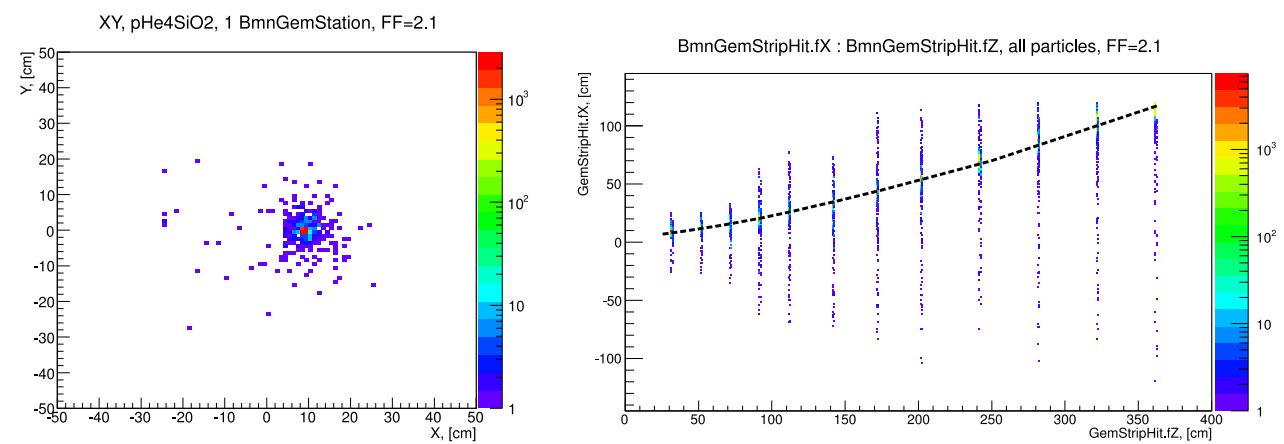

Figure 5. Left panel - the $X Y$ secondary beam profile at the 1-st GEM station for the magnetic field $B_{y}$ of $\sim 0.9 \mathrm{~T}$. Right panel - the $X Z$ profile of the secondary beam hits in the GEM tracker stations for the magnetic field $B_{y}$ of $\sim 0.9$ T. Dashed line represents mean path of scattered particles.

with magnetic elements tuned for the optimal momentum of the secondary protons $\sim 75 \%$ of the initial deuteron beam momentum. The simulation has been performed for the initial deuteron kinetic energy $T_{d}=1.5 \mathrm{GeV}$ and $30 \mathrm{~cm}$ of the ${ }^{3} \mathrm{He}$ target cell using the GEANT4 toolkit. The software model reflects only substantial parts of BM@N setup (start version). In the first variant of simulation ${ }^{3} \mathrm{He}$ target has only been used while in the second one the quartz radiator of $2 \mathrm{~mm}$ thick was added as a TOF start counter after ${ }^{3} \mathrm{He}$ target. The hadronic interaction of the secondary particles with the target, air and start counter was simulated using GEANT4 with using QBBC physics list with the Binary Cascade model. Quartz radiator spreads the size and the number of particles in the secondary beam due to additional multiple scattering in comparison with the first variant, however, the number of these events is quite small for both cases. The $X Y$ secondary beam profile of the first GEM tracker station is presented on the left panel in figure 5. One can see that the $X$-position of the beam is $\sim 10 \mathrm{~cm}$ from the central axis of the spectrometer. The $X Z$ profile of the secondary beam hits in the GEM tracker stations is shown on the right panel in figure 5. The deflection angle is about $\sim 320 \mathrm{mr}$. The time difference between $\mathrm{mRPC}$ and start detectors for protons and deuterons for the base line of $\sim 24 \mathrm{~m}$ are separated by $\sim 20 \mathrm{~ns}$ which is good enough to distinguish between them. The further simulation has been performed for the setup with quartz radiator. The momentum reconstruction of particles is based on the information derived from 12 GEM stations placed in the inhomogeneous magnetic field defined by a field map. The simulation has been performed at different field map scale factors: "half" of the magnetic field value and at maximal one. BM@N setup with 12 stations of GEM tracker situated in the magnet can provide the momentum resolution about few percents. The time difference between mRPC and start detector will be used to separate protons from the background deuterons. Additional improvement of the momentum resolution can be done by removing of the additional material along the beam line as well as by the increasing of the space resolution of the tracking detectors.

\subsection{Other possible reaction at extracted deuteron beam}

The polarization observables in the $p(d, p) d[38,39]$ and $d(d, p) t[41-43]$ reactions in the collinear geometry can be measured using liquid hydrogen or deuterium target or $\mathrm{CH}_{2}$ and $\mathrm{CD}_{2}$ solid targets with Carbon background subtraction. The solid nuclear target can be placed inside the pole of the 3SP40 magnet. It can be varied from the beginning to the middle of the pole of the 3SP40 magnet. This configuration can be used for the measurements of the tensor $A_{y y}$ and vector $A_{y}$ analyzing powers (and, 
possibly, vector polarization transfer coefficient $\left.C_{y}^{y}\right)$ in inclusive deuteron breakup, $A(d, p) X$, at large transverse proton momenta [15] at the highest available energy at Nuclotron; of the tensor $A_{y y}$ and vector $A_{y}$ analyzing powers [44-46] (and, possibly, vector polarization transfer coefficient $C_{y}^{y}$ [47]) in the inelastic deuteron scattering, $A\left(d, d^{\prime}\right) X$, in the vicinity of the baryonic resonances excitation; of the tensor $A_{y y}$ and vector $A_{y}$ analyzing powers in the inclusive pion production, $A\left(d, \pi^{-}\right) X,[48]$ also at the highest available energy at Nuclotron. The measurements of the analyzing powers in inclusive deuteron breakup and the inelastic deuteron scattering in the vicinity of the baryonic resonances excitation can be performed simultaneously. All these experiments require the additional TOF detector placed between F5 and F6 focuses. The separation of the primary deuteron and secondary beams at focus point F6 is provided by the modernized analyzing magnet SP-41 [35]. The physics at F6 is related with the baryonic resonances spin properties studies at the energies between 2 and $6 \mathrm{GeV}$ of the deuteron kinetic energy and includes the measurements of the tensor $A_{y y}$ and vector $A_{y}$ analyzing powers in quasi-elastic and inelastic $A(d, p p) X$ reaction; of the tensor $A_{y y}$ and vector $A_{y}$ analyzing powers in the inelastic deuteron scattering $A\left(d, d^{\prime}\right) X[47]$ and $A\left(d, d^{\prime}\right) \pi^{ \pm} X$ reactions; investigation of the vector analyzing power in neutron induced reactions (with the proton spectator detection) like $n p \rightarrow p n, n p \rightarrow p p \pi^{-}, n p \rightarrow n p \pi^{+} \pi^{-}[49,50]$ etc. For these experiments the full size RPC wall is required. In parallel, the measurement of the $d p$ - elastic scattering [23, 51], $d d \rightarrow{ }^{3} \mathrm{Hen}$ [52-54] and $d A \rightarrow{ }^{3} \mathrm{He} X$ [55] processes can be performed. The spin studies with BM@N require also the advanced deuteron beam polarimetry at Nuclotron discussed in [56, 57].

\section{Deuteron polarization measurement}

Measurements of the deuteron vector and tensor beam polarization were performed at the end of linear accelerator in June-July 2016 with new polarized ion source. Deuteron vector and tensor beam polarization at energy of $10 \mathrm{MeV}$ measured with low energy polarimeter are shown in figure 6 . Red symbols represent the 1-4 transition with ideal values of polarization $\left(p_{z}, p_{z z}\right)=(+1 / 2,-1 / 2)$, blue symbols are for the case of 3-4 transition with ideal values of polarization $\left(p_{z}, p_{z z}\right)=(-1 / 2,-1 / 2)$. The measurement of deuteron polarization with low energy polarimeter shows good values of vector and tensor polarizations.

\section{Conclusion}

$D p$ breakup and $d p$ elastic data along with theoretical description based on relativistic multi-scattering model were discussed. Monte Carlo study of ${ }^{3} \mathrm{He}(d, p)^{4} \mathrm{He}$ reaction at $1.5 \mathrm{GeV}$ confirms suitability of experiment performed on extracted beam. New PIS can provide unique opportunity for the studies of the spin effects and polarization phenomena in few body systems at ITS and also on extracted beam with the BM@N setup. Commissioning of the upgraded DSS setup has been performed at $270 \mathrm{MeV}$ using unpolarized deuteron beam in June-July 2016. The measurement with low energy polarimeter showed good values of vector and tensor polarizations. New data can be measured at ITS at the Nuclotron using new PIS, e.g. data on the analyzing powers $A_{y}, A_{y y}$ and $A_{x x}$ in dp- elastic scattering at various energies up to $2000 \mathrm{MeV}$, and the $d p$-nonmesonic breakup at the energies between 300 and $500 \mathrm{MeV}$ for various kinematic configurations. Proposed experiments on extracted deuteron beam were discussed.

\section{Acknowledgement}

The authors thank P. N. Batyuk, A.D. Kovalenko, L.V. Kutuzova, V.I. Maximenkova, S. P. Merts, Yu.V. Prokofichev, V.B. Shutov, A.V. Turbabin and V.N. Zubets for their help in the preparation of new 

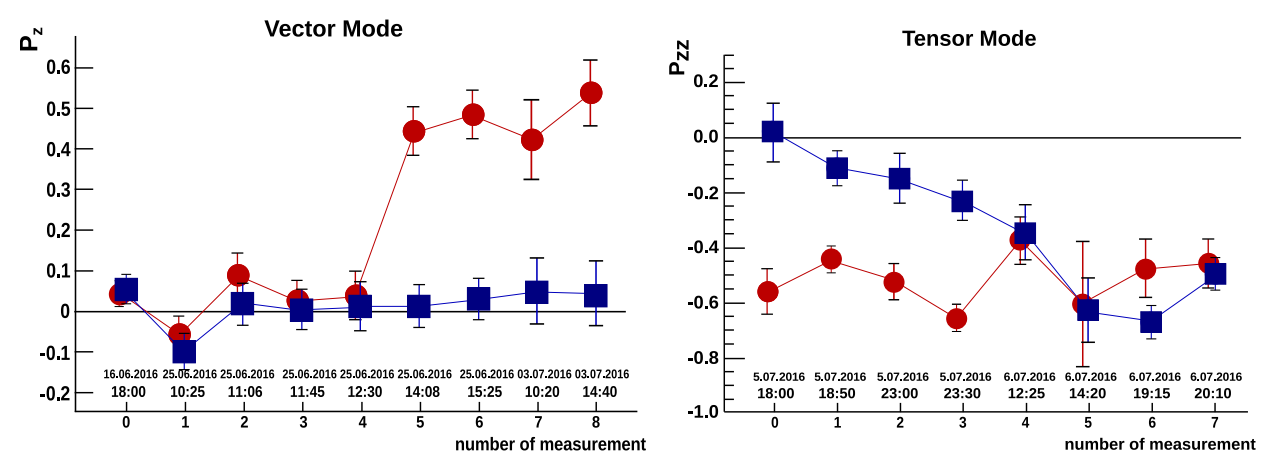

Figure 6. Left panel - deuteron vector beam polarization at energy of $10 \mathrm{MeV}$. Right panel - deuteron tensor beam polarization at energy of $10 \mathrm{MeV}$. Red symbols represent the 1-4 transition with ideal values of polarization $\left(p_{z}, p_{z z}\right)=(+1 / 2,-1 / 2)$, blue symbols are for the case of 3-4 transition with ideal values of polarization $\left(p_{z}, p_{z z}\right)=$ $(-1 / 2,-1 / 2)$.

polarized ion source and experimental setup. The work has been supported in part by the RFBR under grant 16-02-00203a, JINR-Slovak Republic and JINR-Romania Scientific Cooperation programs in 2015-2016 years.

\section{References}

[1] S. A. Coon et al., Nucl. Phys. A 317, 242 (1979)

[2] S. A. Coon, W. Glockle, Phys. Rev. C 23, 1790 (1981)

[3] V. G. J. Stoks, R. A. M. Klomp, C. P. F. Terheggen, J. J. de Swart, Phys. Rev. C 49, 2950 (1994)

[4] P.F. Bedanque, U. Kolck, Ann. Rev. Nucl. Part. Sci. 52, 339 (2002)

[5] H. Setze et al., AIP Conf. Proc. 334, 463 (1995)

[6] W. Tornow et al., Few-Body Syst. Suppl. 8, 163 (1995)

[7] F. Correll et al., Nucl. Phys. A 475, 407 (1998)

[8] G. Rauprich et al., Nucl. Phys. A 535, 313 (1991)

[9] H. Patberg et al., Phys. Rev. C 53, 1497 (1996)

[10] K. Hatanaka, Y. Shimizu et al., Phys.Rev. C 66, 044002 (2002)

[11] N. Sakamoto et al., Phys. Lett. B 367, 60 (1996)

[12] K. Sekiguchi et al., Phys. Rev. C 79, 054008 (2009)

[13] H.O. Meyer et al., Phys. Rev. Lett. 93, 112502 (2004)

[14] S. Kistryn et al., Phys. Rev. C 72, 044006 (2005)

[15] L.S. Azhgirey et al., Phys.Lett. B 387, 37 (1996)

[16] R. Machleidt, Phys.Rev. C 63, 024001 (2001)

[17] V.A. Karmanov and A.V. Smirnov, Nucl.Phys. A 546, 691 (1992)

[18] V.P. Ladygin et al., J.Phys.Conf.Ser. 295, 012131 (2011)

[19] A.I. Malakhov et al., Nucl. Instr. Meth. in Phys. Res. A 440, 320 (2000)

[20] S.M. Piyadin et al., Physics of Particles and Nuclei Letters 8(2), 107 (2011) 
[21] M. Janek et al., Physics of Particles and Nuclei Letters, 11(4), 552 (2014)

[22] H. Witala et al., Few-Body Syst. 49, 61 (2011)

[23] P.K. Kurilkin et al., Phys.Lett. B 715, 61 (2012)

[24] J.C. Alder et al., Phys.Rev. C 6, 2010 (1972)

[25] N.E. Booth et al., Phys.Rev. D 4, 1261 (1971)

[26] W. Glöckle, H. Witala, D. Hüber, H. Kamada and J. Golak, Phys.Rept. 274, 107 (1996)

[27] N.B. Ladygina, Phys.Atom.Nucl. 71, 2039 (2008)

[28] N.B. Ladygina, Eur.Phys.J. A 42, 91 (2009)

[29] M.A. Shikhalev, Phys.Atom.Nucl. 72, 588 (2009)

[30] E. Culmez, Phys.Rev. C 43(5) (1991)

[31] E. Winkelmann et al., Phys.Rev. C 21(6), 2535-2541 (1980)

[32] V.V. Fimushkin et al., Eur. Phys. J. ST 162, 275 (2008)

[33] V. Ladygin et al., PoS(Baldin-ISHEPP-XXI)38 (2012)

[34] S.M. Piyadin et al., Phys. Part. Nucl. Lett. 9, 589 (2012)

[35] P.G. Akishin et al., Phys.Part.Nucl.Lett. 12, 305 (2015)

[36] T. Uesaka et al., Nucl.Instr. and Meth. in Phys.Res. A 402, 212 (1998)

[37] M. Janek et al., Journal of physics: conference series 678, 012032 (2016)

[38] V. Punjabi et al., Phys.Lett. B 350, 178 (1995)

[39] L.S. Azhgirey et al., Phys.Lett. B 391, 22 (1997)

[40] T. Uesaka et al., Phys.Lett. B 467, 199 (1999)

[41] V.P. Ladygin et al., Phys.Lett. B 598, 47 (2004)

[42] N.B. Ladygina, Few Body Syst. 53, 253 (2012)

[43] N.B. Ladygina, Phys.Part.Nucl. 45, 187 (2014)

[44] V.P. Ladygin et al., Eur.Phys.J. A 8, 409 (2000)

[45] L.S. Azhgirey et al., Phys.Atom.Nucl. 68, 991 (2005)

[46] V.P. Ladygin et al., Phys.Atom.Nucl. 69, 852 (2006)

[47] V.P. Ladygin and N.B. Ladygina, Phys.Atom.Nucl. 65, 182 (2002)

[48] L.S. Azhgirey et al., Phys.Atom.Nucl. 74, 1392 (2011)

[49] G. Agakishiev et al., Phys.Lett. B 750, 184 (2015)

[50] A. Jerusalimov et al., Eur.Phys.J. A 51, 83 (2015)

[51] P.K. Kurilkin et al., Phys.Part.Nucl.Lett. 8, 1081 (2011)

[52] M. Janek et al., Eur.Phys.J. A 33, 39 (2007)

[53] A.K. Kurilkin et al., Int.J.Mod.Phys. A 24, 526 (2009)

[54] A.K. Kurilkin et al., Phys.Rev. C 87, 051001 (2013)

[55] V.P. Ladygin and N.B. Ladygina, Phys.Atom.Nucl. 58, 1283 (1995)

[56] P.K. Kurilkin et al., Nucl.Instr.Meth. in Phys.Res. A 642, 45 (2011)

[57] P.K. Kurilkin and V.P. Ladygin, Phys.Part.Nucl. 45, 265 (2014) 